\title{
Existence of nonnegative nontrivial periodic solutions to a doubly degenerate parabolic equation with variable exponent
}

\section{Zhongqing Li ${ }^{*}$ and Wenjie Gao}

$\overline{\text { "Correspondence: zqli_jlu@163.com }}$ College of Mathematics, Jilin University, Changchun, 130012, PR China

\begin{abstract}
The authors investigate a degenerate parabolic equation with delay and nonlocal term, which describes slow diffusive processes in physics or biology. The existence of a nonnegative nontrivial periodic solution is obtained through the use of the Leray-Schauder degree method.

MSC: Primary 35D05; secondary 35K55

Keywords: degenerate parabolic equation; periodic solution; variable exponent; topological degree; De Giorgi iteration
\end{abstract}

\section{Introduction}

In this paper, we are interested in the following evolutional $p(x)$-Laplacian equation:

$$
\begin{cases}\frac{\partial u}{\partial t}-\operatorname{div}\left(\left|\nabla u^{m}\right|^{p(x)-2} \nabla u^{m}\right) & \\ \quad=\left[a(x, t)-\int_{\Omega} K(\xi, t) u^{2}(\xi, t-\tau) d \xi\right] u, & (x, t) \in Q_{T}, \\ u(x, t)=0, & (x, t) \in \Gamma_{T}, \\ u(x, 0)=u(x, T), & x \in \Omega .\end{cases}
$$

Here, $\Omega$ is a bounded simply connected domain with smooth boundary $\partial \Omega$ in $\mathbb{R}^{N}, Q_{T}=$ $\Omega \times(0, T), \Gamma_{T}=\partial \Omega \times(0, T), T>0$, and $\tau \in(0,+\infty)$. We assume $m>1, p \in C^{1, \alpha}(\bar{\Omega})$, with $p^{+}:=\max _{\bar{\Omega}} p(x), p^{-}:=\min _{\bar{\Omega}} p(x), p^{+} \geq p^{-}>2$ and that $a \in L^{\infty}\left(Q_{T}\right)$ and $K \in L^{\infty}\left(Q_{T}\right)$ can be extended as $T$-periodic functions to $\Omega \times R$. Furthermore, we assume that $K \geq 0$ for a.e. $(x, t) \in Q_{T}$.

Equation (1.1) is a doubly degenerate parabolic equation with delay and nonlocal term, which models diffusive periodic phenomena in physics and mathematical biology. In biology, it arises from population model, where $u(x, t)$ denotes the density of population at time $t$ located at $x \in \Omega, a$ is the natural growth rate of the population, the nonlocal term $\int_{\Omega} K(\xi, t) u^{2}(\xi, t-\tau) d \xi$ evaluates a weighted fraction of individual, and the delayed density $u$ at time $t-\tau$ appearing in the nonlocal term represents the time needed to an individual to become adult. In physics, problem (1.1) is proposed based on some evolution phenomena in electrorheological fluids [1]. It describes the ability of a conductor to undergo significant changes when an electric field is imposed on. This model has been 
employed for some technological applications, such as medical rehabilitation equipment and shock wave absorber.

When $p(x)$ is a constant and $m>1, p>2$, the model describes the slow diffusion process in physics, which has been extensively investigated; see [2-7]. For example, in [5], the authors studied the following doubly degenerate parabolic equation with logistic periodic sources:

$$
\frac{\partial u}{\partial t}-\operatorname{div}\left(\left|\nabla u^{m}\right|^{p-2} \nabla u^{m}\right)=u^{\alpha}\left(a-b u^{\beta}\right) .
$$

They proved the existence of a nontrivial nonnegative periodic solution via monotonicity method. Using a Moser iterative method (see [8-11]), they also obtained some a priori bounds and asymptotic behaviors for the solutions.

Recently, the variable exponent Sobolev space and its applications have attracted considerable interest; see [1,12-14] and the references therein. When $p^{+} \geq p^{-}>2$ and $m>1$, the doubly degenerate parabolic equation (1.1) is a more realistic model which describes the rather slow diffusion process. In our models, the principal term $\operatorname{div}\left(\left|\nabla u^{m}\right|^{p(x)-2} \nabla u^{m}\right)$, in place of the usual term $\Delta u^{m}$ or $\operatorname{div}\left(\left|\nabla u^{m}\right|^{p-2} \nabla u^{m}\right)$, represents nonhomogeneous diffusion that depends on the position $x \in \Omega$ and thus gives a better description of nonhomogeneous character of the process.

There are many differences between Sobolev spaces with constant exponent and those with variable exponent; many powerful tools applicable in constant exponent spaces are not available for variable exponent spaces. For instance, the variable exponent spaces are no longer translation invariant and Young's inequality $\|f * g\|_{p(\cdot)} \leq C\|f\|_{p(\cdot)}\|g\|_{1}$ holds if and only if $p$ is constant (see monograph [12]). As we all know, the frequently used Hölder's inequality, Poincarés inequality, etc., will be presented in new forms for variable exponent spaces.

The presence of the nonlocal term and $p(x)$-Laplacian term makes the sup-solution and sub-solution method (as in [5]) in vain. In our paper, we adopt the topological degree method (as in [8-10]) to show the existence of nontrivial periodic solutions to problem (1.1). However, the method employed in the variable exponent case [13] or in the constant exponent case [8-11] cannot be directly used to derive the uniform upper bound for solutions, which is a crucial step in applying the topological degree method. We apply a modified De Giorgi iteration to establish the crucial uniform bound. We believe that the modified De Giorgi iteration used in this paper can be employed to other types equations with nonstandard growth conditions.

We now discuss the main plan of the paper. In Section 2, we review some preliminaries concerning the variable exponent Sobolev spaces and introduce a family of regularized problems for problem (1.1). We regularize the degenerate part through replacing the term $\operatorname{div}\left(\left|\nabla u^{m}\right|^{p(x)-2} \nabla u^{m}\right)$ by

$$
\operatorname{div}\left\{\left(\left|\nabla\left(\sigma u^{m}+\epsilon u\right)\right|^{2}+\eta\right)^{\frac{p(x)-2}{2}} \nabla\left(\sigma u^{m}+\epsilon u\right)\right\}, \quad \epsilon, \eta>0 .
$$

In Section 3, in order to apply the topological degree method, we combine these regularized problems with a relatively simpler equation and derive some a priori estimates. By virtue of the De Giorgi iteration technique, we deduce an a priori $L^{\infty}$ bound for solutions to the regularized problems in Proposition 3.2; and the uniform lower bound estimate is obtained in Proposition 3.5. In Section 4, we establish the existence of nonnegative non- 
trivial solution of (1.1) through the limit process as $\epsilon$ and $\eta$ tend to zero. Finally, in the Appendix, we give a proof of the iteration lemma (Lemma 3.1) for the sake of readability.

\section{Preliminaries and the regularized problems of (1.1)}

First of all, for the reader's convenience, we recall some preliminary results concerning the variable exponent Sobolev spaces. One may find these standard results in monographs [1, 12].

Let $p$ be a continuous function defined in $\bar{\Omega}, p(x)>1$, for any $x \in \bar{\Omega}$.

1. $L^{p(x)}(\Omega)$ space: We have

$$
L^{p(x)}(\Omega):=\left\{u: u \text { is measurable in } \Omega \text { and } \int_{\Omega}|u(x)|^{p(x)} d x<\infty\right\},
$$

equipped with the following Luxemburg norm:

$$
|u|_{L^{p(x)}(\Omega)}:=\inf \left\{\lambda>0: \int_{\Omega}\left|\frac{u(x)}{\lambda}\right|^{p(x)} d x \leq 1\right\} .
$$

The space $\left(L^{p(x)}(\Omega),|\cdot|_{L^{p(x)}(\Omega)}\right)$ is a separable, uniformly convex Banach space.

2. $W^{1, p(x)}(\Omega)$ space: We have

$$
W^{1, p(x)}(\Omega):=\left\{u \in L^{p(x)}(\Omega):|\nabla u| \in L^{p(x)}(\Omega)\right\}
$$

endowed with the norm $|u|_{W^{1, p(x)(\Omega)}}:=|\nabla u|_{L^{p(x)}(\Omega)}+|u|_{L^{p(x)}(\Omega)}$. We denote by $W_{0}^{1, p(x)}(\Omega)$ the closure of $C_{0}^{\infty}(\Omega)$ in $W^{1, p(x)}(\Omega)$. In fact, the norm $|\nabla u|_{L^{p(x)}(\Omega)}$ and $|u|_{W^{1, p(x)}(\Omega)}$ are equivalent norms in $W_{0}^{1, p(x)}(\Omega)$. $W^{1, p(x)}(\Omega)$ and $W_{0}^{1, p(x)}(\Omega)$ are separable and reflexive Banach spaces with the above norms.

3. Frequently used relationships in the estimate:

$$
\begin{aligned}
& \min \left\{\left(\int_{\Omega}|u(x)|^{p(x)} d x\right)^{\frac{1}{p^{+}}},\left(\int_{\Omega}|u(x)|^{p(x)} d x\right)^{\frac{1}{p^{-}}}\right\} \\
& \quad \leq|u|_{L^{p(x)}(\Omega)} \\
& \quad \leq \max \left\{\left(\int_{\Omega}|u(x)|^{p(x)} d x\right)^{\frac{1}{p^{+}}},\left(\int_{\Omega}|u(x)|^{p(x)} d x\right)^{\frac{1}{p^{-}}}\right\} .
\end{aligned}
$$

4. $p(x)$-Hölder's inequality:

For any $u \in L^{p(x)}(\Omega)$ and $v \in L^{q(x)}(\Omega)$, with $\frac{1}{p(x)}+\frac{1}{q(x)}=1$, we have

$$
\left|\int_{\Omega} u v d x\right| \leq\left(\frac{1}{p^{-}}+\frac{1}{q^{-}}\right)|u|_{L^{p(x)}(\Omega)}|v|_{L^{q(x)}(\Omega)} .
$$

\section{Embedding relationships:}

If $p_{1}$ and $p_{2}$ are in $C(\bar{\Omega})$, and $1 \leq p_{1}(x) \leq p_{2}(x)$, for any $x \in \bar{\Omega}$, then there exists a positive constant $C_{p_{1}(x), p_{2}(x)}$ such that

$$
|u|_{L^{p_{1}(x)}(\Omega)} \leq C_{p_{1}(x), p_{2}(x)}|u|_{L^{p_{2}(x)}(\Omega)}
$$

i.e. the embedding $L^{p_{2}(x)}(\Omega) \hookrightarrow L^{p_{1}(x)}(\Omega)$ is continuous. 
If $q \in C(\bar{\Omega})$ and $1 \leq q(x)<p^{*}(x)$, for any $x \in \bar{\Omega}$, then the embedding $W_{0}^{1, p(x)}(\Omega) \hookrightarrow$ $L^{q(x)}(\Omega)$ is continuous and compact. Here

$$
p^{*}(x):= \begin{cases}\frac{N p(x)}{N-p(x)}, & p(x)<N \\ +\infty, & p(x) \geq N\end{cases}
$$

6. $p(x)$-Poincarés inequality:

There exists a positive constant $C_{p}$ such that $|u|_{L^{p(x)}(\Omega)} \leq C_{p}|\nabla u|_{L^{p(x)}(\Omega)}$, for any $u \in$ $W_{0}^{1, p(x)}(\Omega)$.

We next define the weak solutions to problem (1.1).

Definition $2.1 u$ is said to be a weak periodic solution to (1.1) provided that $u^{m} \in$ $L^{p^{-}}\left(0, T ; W_{0}^{1, p(x)}(\Omega)\right)$ with $\left|\nabla u^{m}\right| \in L^{p(x)}\left(Q_{T}\right), u \in C\left(\bar{Q}_{T}\right)$ and $u$ satisfies

$$
\begin{aligned}
0= & \iint_{Q_{T}}\left\{-u \frac{\partial \varphi}{\partial t}+\left|\nabla u^{m}\right|^{p(x)-2} \nabla u^{m} \nabla \varphi\right. \\
& \left.-a u \varphi+u \varphi \int_{\Omega} K(\xi, t) u^{2}(\xi, t-\tau) d \xi\right\} d x d t,
\end{aligned}
$$

for all $\varphi \in C^{1}\left(\bar{Q}_{T}\right)$ satisfying $\varphi(x, T)=\varphi(x, 0)$ for $x \in \Omega$ and $\left.\varphi(\cdot, t)\right|_{\partial \Omega}=0$ for $t \in[0, T]$.

As in [7], we introduce the following regularized problem:

$$
\begin{cases}\frac{\partial u}{\partial t}-\operatorname{div}\left\{\left(\left|\nabla\left(\sigma u^{m}+\epsilon u\right)\right|^{2}+\eta\right)^{\frac{p(x)-2}{2}} \nabla\left(\sigma u^{m}+\epsilon u\right)\right\} & \\ \quad=\left[a-\int_{\Omega} K(\xi, t) u^{2}(\xi, t-\tau) d \xi\right] u, & \text { a.e. }(x, t) \in Q_{T}, \\ \left.u(\cdot, t)\right|_{\partial \Omega}=0, & \text { a.e. } t \in(0, T), \\ u(\cdot, 0)=u(\cdot, T), & \end{cases}
$$

where $0<\epsilon<\frac{1}{2}, 0<\eta<\left(\frac{1}{2}\right)^{\frac{p^{+}-2}{p^{-}-2}}$ and $\sigma \in[0,1]$ are given constants.

Definition 2.2 We say that $u_{\epsilon \eta}$ is a weak periodic solution of (2.2), if $u_{\epsilon \eta}^{m} \in L^{p^{-}}(0, T$; $\left.W_{0}^{1, p(x)}(\Omega)\right)$ with $\left|\nabla u_{\epsilon \eta}^{m}\right| \in L^{p(x)}\left(Q_{T}\right), u_{\epsilon \eta} \in C\left(\bar{Q}_{T}\right)$, and $u_{\epsilon \eta}$ solves

$$
\begin{aligned}
0= & \iint_{Q_{T}}\left\{-u_{\epsilon \eta} \frac{\partial \varphi}{\partial t}+\left(\left|\nabla\left(\sigma u_{\epsilon \eta}^{m}+\epsilon u_{\epsilon \eta}\right)\right|^{2}+\eta\right)^{\frac{p(x)-2}{2}} \nabla\left(\sigma u_{\epsilon \eta}^{m}+\epsilon u_{\epsilon \eta}\right) \nabla \varphi\right. \\
& \left.-a u_{\epsilon \eta} \varphi+u_{\epsilon \eta} \varphi \int_{\Omega} K(\xi, t) u_{\epsilon \eta}^{2}(\xi, t-\tau) d \xi\right\} d x d t,
\end{aligned}
$$

for all $\varphi \in C^{1}\left(\bar{Q}_{T}\right)$ satisfying $\varphi(x, T)=\varphi(x, 0)$ for $x \in \Omega$ and $\left.\varphi(\cdot, t)\right|_{\partial \Omega}=0$ for $t \in[0, T]$.

Remark 2.3 For any $p \in C^{1, \alpha}(\bar{\Omega})$, the set $\left\{\varphi \in C^{1}\left(\bar{Q}_{T}\right): \varphi(\cdot, T)=\varphi(\cdot, 0)\right\}$ is dense in $\{\varphi \in$ $\left.C\left(\bar{Q}_{T}\right) \cap L^{p^{-}}\left(0, T ; W_{0}^{1, p(x)}(\Omega)\right):\left|\nabla u^{m}\right| \in L^{p(x)}\left(Q_{T}\right), \varphi(\cdot, T)=\varphi(\cdot, 0)\right\}$, thus in the sense of the definition of weak solution above, $u_{\epsilon \eta}$ can be chosen as test function. 
We investigate problem (2.2) extensively before studying the limit process as $\epsilon, \eta \rightarrow 0$. Define a map $G_{\epsilon \eta}:[0,1] \times L^{\infty}\left(Q_{T}\right) \rightarrow L^{\infty}\left(Q_{T}\right)$ as follows:

$$
(\sigma, f) \mapsto u_{\epsilon \eta}=G_{\epsilon \eta}(\sigma, f),
$$

where $u_{\epsilon \eta}$ is a weak periodic solution of the problem:

$$
\begin{cases}\frac{\partial u}{\partial t}-\operatorname{div}\left\{\left(\left|\nabla\left(\sigma u^{m}+\epsilon u\right)\right|^{2}+\eta\right) \frac{p(x)-2}{2} \nabla\left(\sigma u^{m}+\epsilon u\right)\right\}=f, & \text { a.e. }(x, t) \in Q_{T}, \\ \left.u(\cdot, t)\right|_{\partial \Omega}=0, & \text { a.e. } t \in(0, T), \\ u(\cdot, 0)=u(\cdot, T) . & \end{cases}
$$

Given $\alpha \in L^{\infty}\left(Q_{T}\right)$, let $f=f(\alpha) \in L^{\infty}\left(Q_{T}\right)$ be defined by

$$
f(x, t)=f(\alpha)(x, t)=\left[a(x, t)-\int_{\Omega} K(\xi, t) \alpha^{2}(\xi, t-\tau) d \xi\right] \alpha \quad \text { for }(x, t) \in Q_{T} .
$$

Therefore, if a nonnegative function $u_{\epsilon \eta} \in L^{\infty}\left(Q_{T}\right)$ satisfies $u_{\epsilon \eta}=G_{\epsilon \eta}\left(1, f\left(u_{\epsilon \eta}\right)\right)$, then $u_{\epsilon \eta}$ is a weak solution of (2.2).

Define

$$
T_{\epsilon \eta}(\sigma, u)=G_{\epsilon \eta}(\sigma, f(u)), \quad(\sigma, u) \in[0,1] \times L^{\infty}\left(Q_{T}\right) .
$$

Then, according to [3] or the classical regularity results from [4], one obtains the following lemma.

Lemma 2.4 Assume that $0<\epsilon<\frac{1}{2}, 0<\eta<\left(\frac{1}{2}\right)^{\frac{p^{+}-2}{p^{-}-2}}$ and $\lambda \in L^{\infty}\left(Q_{T}\right)$. Then $T_{\epsilon \eta}$ is a continuous compact operator from $[0,1] \times L^{\infty}\left(Q_{T}\right)$ to $L^{\infty}\left(Q_{T}\right)$. Furthermore, $u_{\epsilon \eta}=T_{\epsilon \eta}(\sigma, \lambda) \in$ $C\left(\bar{Q}_{T}\right)$.

\section{A priori estimates to the regularized problem}

First of all, the following modified De Giorgi iteration lemma will be useful (we give a proof in the Appendix).

Lemma 3.1 (Iteration lemma) Suppose $\varphi(t)$ is a nonnegative and nonincreasing function on $\left[K_{0},+\infty\right)$, it satisfies

$$
\varphi(h) \leq\left(\frac{M}{h-k}\right)^{\alpha}\left[\varphi^{\beta}(k)+\varphi^{\gamma}(k)\right]
$$

for any $h>k \geq k_{0}$, and for some constants $M>0, \alpha>0, \beta>1, \gamma>1$. Then

$$
\varphi\left(k_{0}+d\right)=0
$$

where $d=M 2^{\frac{\delta}{\delta-1}}\left(\varphi^{\beta-1}\left(k_{0}\right)+\varphi^{\gamma-1}\left(k_{0}\right)\right)^{\frac{1}{\alpha}}$, and $\delta=\min \{\beta, \gamma\}$.

Next, we prove a crucial a priori $L^{\infty}$ bound for $u_{\epsilon \eta}$ via a De Giorgi iteration technique as in [15]. 
Proposition 3.2 Let $K_{1}>0$ and assume that $u_{\epsilon \eta}$ is a nonnegative T-periodic continuous function such that

$$
\begin{aligned}
& \frac{\partial u}{\partial t}-\operatorname{div}\left\{\left(\left|\nabla\left(u^{m}+\epsilon u\right)\right|^{2}+\eta\right)^{\frac{p(x)-2}{2}} \nabla\left(u^{m}+\epsilon u\right)\right\} \leq K_{1} u, \\
& \left.u(\cdot, t)\right|_{\partial \Omega}=0 .
\end{aligned}
$$

Then there exists a constant $R>0$, such that $\left\|u_{\epsilon \eta}\right\|_{L^{\infty}\left(Q_{T}\right)}<R$, where $R$ is independent of $\epsilon$ and $\eta$.

Proof Step 1 . Multiplying (3.2) by $u_{\epsilon \eta}^{m q}$, with any $q>1$. Integrating over $\Omega$ and noticing that $\left.u_{\epsilon \eta}(\cdot, t)\right|_{\partial \Omega}=0$, we have

$$
\begin{aligned}
& \frac{1}{m q+1} \frac{d}{d t} \int_{\Omega} u_{\epsilon \eta}^{m q+1} d x \\
& \quad+\int_{\Omega}\left(\left|\nabla\left(u_{\epsilon \eta}^{m}+\epsilon u_{\epsilon \eta}\right)\right|^{2}+\eta\right)^{\frac{p(x)-2}{2}} \nabla\left(u_{\epsilon \eta}^{m}+\epsilon u_{\epsilon \eta}\right) \nabla u_{\epsilon \eta}^{m q} d x \\
& \leq K_{1} \int_{\Omega} u_{\epsilon \eta}^{m q+1} d x .
\end{aligned}
$$

Since $\left|\nabla u_{\epsilon \eta}^{m}\right|^{p(x)} \geq\left|\nabla u_{\epsilon \eta}^{m}\right|^{p^{-}}-1$, we deal with the second term on the left-hand side of (3.4) as follows.

$$
\begin{aligned}
& \int_{\Omega}\left(\left|\nabla\left(u_{\epsilon \eta}^{m}+\epsilon u_{\epsilon \eta}\right)\right|^{2}+\eta\right)^{\frac{p(x)-2}{2}} \nabla\left(u_{\epsilon \eta}^{m}+\epsilon u_{\epsilon \eta}\right) \nabla u_{\epsilon \eta}^{m q} d x \\
& \quad \geq q \int_{\Omega} u_{\epsilon \eta}^{m(q-1)}\left|\nabla u_{\epsilon \eta}^{m}\right|^{p(x)} d x \\
& \quad \geq q \int_{\Omega} u_{\epsilon \eta}^{m(q-1)}\left|\nabla u_{\epsilon \eta}^{m}\right|^{p^{-}} d x-q \int_{\Omega} u_{\epsilon \eta}^{m(q-1)} d x \\
& \quad=q\left(\frac{p^{-}}{p^{-}+q-1}\right)^{p^{-}} \int_{\Omega}\left|\nabla u_{\epsilon \eta}^{\frac{m\left(p^{-}+q-1\right)}{p^{-}}}\right|^{p^{-}} d x-q \int_{\Omega} u_{\epsilon \eta}^{m(q-1)} d x .
\end{aligned}
$$

Combining (3.4) and (3.5), we have

$$
\begin{aligned}
& \frac{1}{m q+1} \frac{d}{d t} \int_{\Omega} u_{\epsilon \eta}^{m q+1} d x+q\left(\frac{p^{-}}{p^{-}+q-1}\right)^{p^{-}} \int_{\Omega}\left|\nabla u_{\epsilon \eta}^{\frac{m\left(p^{-}+q-1\right)}{p^{-}}}\right|^{p^{-}} d x \\
& \quad \leq K_{1} \int_{\Omega} u_{\epsilon \eta}^{m q+1} d x+q \int_{\Omega} u_{\epsilon \eta}^{m(q-1)} d x .
\end{aligned}
$$

We estimate the right-hand side of (3.6) by Hölder's inequality, the embedding theorem and Young's inequality with $\epsilon$ to deduce

$$
\begin{aligned}
K_{1} \int_{\Omega} u_{\epsilon \eta}^{m q+1} d x+q \int_{\Omega} u_{\epsilon \eta}^{m(q-1)} d x \\
\leq K_{1}\left(\int_{\Omega} u_{\epsilon \eta}^{m\left(p^{-}+q-1\right)} d x\right)^{\frac{m q+1}{m\left(p^{-+}+-1\right)}}|\Omega|^{1-\frac{m q+1}{m\left(p^{-}+q-1\right)}} \\
\quad+q\left(\int_{\Omega} u_{\epsilon \eta}^{m\left(p^{-}+q-1\right)} d x\right)^{\frac{q-1}{p^{-+q-1}}}|\Omega|^{1-\frac{q-1}{p^{-+q-1}}}
\end{aligned}
$$




$$
\begin{aligned}
\leq & C\left(\int_{\Omega}\left|\nabla u_{\epsilon \eta}^{\frac{m\left(p^{-}+q-1\right)}{p^{-}}}\right|^{p^{-}} d x\right)^{\frac{m q+1}{m\left(p^{-}+q-1\right)}} \\
& +C\left(\int_{\Omega}\left|\nabla u_{\epsilon \eta}^{\frac{m\left(p^{-}+q-1\right)}{p^{-}}}\right|^{p^{-}} d x\right)^{\frac{q-1}{p^{-}+q-1}} \\
\leq & \epsilon_{1} \int_{\Omega}\left|\nabla u_{\epsilon \eta}^{\frac{m\left(p^{-}+q-1\right)}{p^{-}}}\right|^{p^{-}} d x+C\left(\epsilon_{1}\right) C^{\frac{m\left(p^{-}+q-1\right)}{m p^{-}-m-1}} \\
& +\epsilon_{2} \int_{\Omega}\left|\nabla u_{\epsilon \eta}^{\frac{m\left(p^{-}+q-1\right)}{p^{-}}}\right|^{p^{-}} d x+C\left(\epsilon_{2}\right) C^{\frac{p^{-}+q-1}{p^{-}}} .
\end{aligned}
$$

Choosing $\epsilon_{1}$ and $\epsilon_{2}$ appropriately, we have from (3.6) and (3.7)

$$
\frac{d}{d t} \int_{\Omega} u_{\epsilon \eta}^{m q+1}(x, t) d x \leq C_{1}
$$

for any $q>1$, where $C_{1}$ depends on $q, p^{-}, m$, and $\Omega$.

Integrating (3.6) over $[\tau, \tau+T]$ and using the $T$-periodicity of $u_{\epsilon \eta}$, we have

$$
\begin{aligned}
& q\left(\frac{p^{-}}{p^{-}+q-1}\right)^{p^{-}} \iint_{Q_{T}}\left|\nabla u_{\epsilon \eta}^{\frac{m\left(p^{-}+q-1\right)}{p^{-}}}\right|^{p^{-}} d x d t \\
& \quad \leq K_{1} \iint_{Q_{T}} u_{\epsilon \eta}^{m q+1} d x d t+q \iint_{Q_{T}} u_{\epsilon \eta}^{m(q-1)} d x d t .
\end{aligned}
$$

Similarly to (3.7), we obtain

$$
\iint_{Q_{T}}\left|\nabla u_{\epsilon \eta}^{\frac{m\left(p^{-}+q-1\right)}{p^{-}}}\right|^{p^{-}} d x d t \leq C_{2}
$$

where $C_{2}$ depends on $q, p^{-}, m, T$ and $\Omega$. By Poincaré's inequality, we have

$$
\iint_{Q_{T}} u_{\epsilon \eta}^{m\left(p^{-}+q-1\right)} d x d t \leq \iint_{Q_{T}}\left|\nabla u_{\epsilon \eta}^{\frac{m\left(p^{-}+q-1\right)}{p^{-}}}\right|^{p^{-}} d x d t \leq C_{2} .
$$

Recall our assumption that $p^{-}>2, m>1$, and thus $m\left(p^{-}+q-1\right)>m q+1$. Consequently, considering (3.11), we obtain

$$
\iint_{Q_{T}} u_{\epsilon \eta}^{m q+1}(x, t) d x d t \leq C_{2}
$$

which implies that there exists a $t_{0} \in(\tau, \tau+T)$ such that

$$
\int_{\Omega} u_{\epsilon \eta}^{m q+1}\left(x, t_{0}\right) d x \leq C_{2} \text {. }
$$

From (3.8) and (3.13), we conclude

$$
\int_{\Omega} u_{\epsilon \eta}^{m q+1}(x, t) d x \leq C_{2}+C_{1}\left(t-t_{0}\right)
$$

for any $t \geq t_{0}$. In view of the $T$-periodicity of $u_{\epsilon \eta}$, (3.14) shows

$$
\int_{\Omega} u_{\epsilon \eta}^{m q+1}(x, \tau) d x=\int_{\Omega} u_{\epsilon \eta}^{m q+1}(x, \tau+T) d x \leq C_{2}+C_{1} T .
$$


We finally arrive at

$$
\sup _{t \in(\tau, \tau+T)} \int_{\Omega} u_{\epsilon \eta}^{m q+1}(x, t) d x \leq C,
$$

for any $q>1$, where $C$ depends on $q, p^{-}, m, T$ and $\Omega$.

Step 2. Let

$$
A_{k}(t)=\left\{x \in \Omega ; u_{\epsilon \eta}(x, t)>k\right\}, \quad \mu_{k}=\sup _{t \in(\tau, \tau+T)}\left|A_{k}(t)\right|,
$$

where $\left|A_{k}(t)\right|$ is the Lebesgue measure of the set $A_{k}(t)$. Multiplying (3.2) by $\left(u_{\epsilon \eta}-\right.$ $k)_{+}^{m} \chi_{\left[t_{1}, t_{2}\right]}(t)$ on both sides, where $\chi_{\left[t_{1}, t_{2}\right]}(t)$ represents the characteristic function of the interval $\left[t_{1}, t_{2}\right]$, and integrating over $Q_{T}$, we have

$$
\begin{aligned}
\frac{1}{m+1} & \int_{t_{1}}^{t_{2}} \frac{d}{d t} \int_{\Omega}\left(u_{\epsilon \eta}-k\right)_{+}^{m+1} d x d t \\
& +\int_{t_{1}}^{t_{2}} \int_{\Omega}\left(\left|\nabla\left(u_{\epsilon \eta}^{m}+\epsilon u_{\epsilon \eta}\right)\right|^{2}+\eta\right)^{\frac{p(x)-2}{2}} \nabla\left(u_{\epsilon \eta}^{m}+\epsilon u_{\epsilon \eta}\right) \nabla\left(u_{\epsilon \eta}-k\right)_{+}^{m} d x d t \\
\leq & K_{1} \int_{t_{1}}^{t_{2}} \int_{\Omega} u_{\epsilon \eta}\left(u_{\epsilon \eta}-k\right)_{+}^{m} d x d t .
\end{aligned}
$$

Let $I_{k}(t):=\int_{\Omega}\left(u_{\epsilon \eta}-k\right)_{+}^{m+1} d x$. We assume that the absolutely continuous function $I_{k}(t)$ attains its maximum at $\varrho \in[\tau, \tau+T]$. Take $t_{1}=\varrho-\theta, t_{2}=\varrho$ and $\theta$ small enough so that $t_{1}>\tau$. (In fact, this is always possible because of the periodicity of $u_{\epsilon \eta}$; for example, if $\varrho=\tau$, we take $\varrho=\tau+T$, then $\varrho>\tau$ and $t_{1}>\tau$.) Then we have $I_{k}(\varrho) \geq I_{k}(\varrho-\theta)$ and

$$
\begin{aligned}
& \frac{1}{\theta} \int_{\varrho-\theta}^{\varrho} \int_{\Omega}\left(\left|\nabla\left(u_{\epsilon \eta}^{m}+\epsilon u_{\epsilon \eta}\right)\right|^{2}+\eta\right)^{\frac{p(x)-2}{2}} \nabla\left(u_{\epsilon \eta}^{m}+\epsilon u_{\epsilon \eta}\right) \nabla\left(u_{\epsilon \eta}-k\right)_{+}^{m} d x d t \\
& \quad \leq \frac{1}{\theta} K_{1} \int_{\varrho-\theta}^{\varrho} \int_{\Omega} u_{\epsilon \eta}\left(u_{\epsilon \eta}-k\right)_{+}^{m} d x d t .
\end{aligned}
$$

Letting $\theta \rightarrow 0^{+}$yields

$$
\begin{aligned}
& \int_{\Omega}\left(\left|\nabla\left(u_{\epsilon \eta}^{m}+\epsilon u_{\epsilon \eta}\right)\right|^{2}+\eta\right)^{\frac{p(x)-2}{2}} \nabla\left(u_{\epsilon \eta}^{m}+\epsilon u_{\epsilon \eta}\right) \nabla\left(u_{\epsilon \eta}-k\right)_{+}^{m} d x \\
& \quad \leq K_{1} \int_{\Omega} u_{\epsilon \eta}\left(u_{\epsilon \eta}-k\right)_{+}^{m} d x .
\end{aligned}
$$

After a direct computation, we obtain an estimate for the left-hand side of (3.17) as follows:

$$
\begin{aligned}
& \int_{\Omega}\left(\left|\nabla\left(u_{\epsilon \eta}^{m}+\epsilon u_{\epsilon \eta}\right)\right|^{2}+\eta\right)^{\frac{p(x)-2}{2}} \nabla\left(u_{\epsilon \eta}^{m}+\epsilon u_{\epsilon \eta}\right) \nabla\left(u_{\epsilon \eta}-k\right)_{+}^{m} d x \\
& \geq \int_{\Omega}\left|m\left(u_{\epsilon \eta}-k\right)_{+}^{m-1} \nabla\left(u_{\epsilon \eta}-k\right)_{+}\right|^{p(x)} d x \\
& \quad=\int_{\Omega}\left|\nabla\left(u_{\epsilon \eta}-k\right)_{+}^{m}\right|^{p(x)} d x \\
& \geq \int_{A_{k}(\varrho)}\left|\nabla\left(u_{\epsilon \eta}-k\right)^{m}\right|^{p^{-}} d x-\left|A_{k}(\varrho)\right| .
\end{aligned}
$$


Substituting (3.18) into (3.17), we have

$$
\int_{A_{k}(\varrho)}\left|\nabla\left(u_{\epsilon \eta}-k\right)^{m}\right|^{p^{-}} d x \leq K_{1} \int_{A_{k}(\varrho)} u_{\epsilon \eta}\left(u_{\epsilon \eta}-k\right)^{m} d x+\mu_{k}
$$

We now deal with (3.19). On one hand, by the embedding theorem

$$
\left(\int_{A_{k}(\varrho)}\left(u_{\epsilon \eta}-k\right)^{m r} d x\right)^{\frac{p^{-}}{r}} \leq S^{p^{-}} \int_{A_{k}(\varrho)}\left|\nabla\left(u_{\epsilon \eta}-k\right)^{m}\right|^{p^{-}} d x
$$

where $S$ is the Sobolev embedding constant, and

$$
r= \begin{cases}\frac{N p^{-}}{N-p^{-}}, & \text {if } p^{-}<N \\ \frac{2 p^{-}\left(N+p^{-}\right)}{N}, & \text { if } p^{-} \geq N .\end{cases}
$$

On the other hand, from (3.15), where we may fix a special $q$, using Hölder's inequality, we obtain

$$
\begin{aligned}
& K_{1} \int_{A_{k}(\varrho)} u_{\epsilon \eta}\left(u_{\epsilon \eta}-k\right)^{m} d x \\
& \quad \leq K_{1}\left(\int_{A_{k}(\varrho)} u_{\epsilon \eta}^{\frac{N+p^{-}}{p^{-}}} d x\right)^{\frac{p^{-}}{N+p^{-}}}\left(\int_{A_{k}(\varrho)}\left(u_{\epsilon \eta}-k\right)^{m \frac{N+p^{-}}{N}} d x\right)^{\frac{N}{N+p^{-}}} \\
& \quad \leq C\left(\int_{A_{k}(\varrho)}\left(u_{\epsilon \eta}-k\right)^{m \frac{N+p^{-}}{N}} d x\right)^{\frac{N}{N+p^{-}}} \\
& \quad \leq C\left|A_{k}(\varrho)\right|^{\frac{N r-N-p^{-}}{r\left(N+p^{-}\right)}}\left(\int_{A_{k}(\varrho)}\left(u_{\epsilon \eta}-k\right)^{m r} d x\right)^{\frac{1}{r}} .
\end{aligned}
$$

Let $J_{k}(\varrho)=\int_{A_{k}(\varrho)}\left(u_{\epsilon \eta}-k\right)^{m r} d x$. Then (3.19), (3.20), and (3.21) imply

$$
\frac{1}{S^{p^{-}}}\left[J_{k}(\varrho)\right]^{\frac{p^{-}}{r}} \leq C \mu_{k}^{\frac{N r-N-p^{-}}{r\left(N+p^{-}\right)}}\left[J_{k}(\varrho)\right]^{\frac{1}{r}}+\mu_{k}
$$

Utilizing Young's inequality with $\epsilon$, we obtain from (3.22)

$$
\begin{aligned}
J_{k} & \leq 2^{\frac{r}{p^{-}}}\left(S^{r} C^{\frac{r}{p^{-}}} \mu_{k}^{\frac{N r-N-p^{-}}{p^{-}\left(N+p^{-}\right)}} J_{k}^{\frac{1}{p^{-}}}+S^{r} \mu_{k}^{\frac{r}{p^{-}}}\right) \\
& \leq C \mu_{k}^{\frac{N r-N-p^{-}}{p^{-}\left(N+p^{-}\right)}} J_{k}^{\frac{1}{p^{-}}}+C \mu_{k}^{\frac{r}{p^{-}}} \\
& \leq C\left(\epsilon J_{k}+C(\epsilon) \mu_{k}^{\frac{N r-N-p^{-}}{\left(p^{-}-1\right)\left(N+p^{-}\right)}}\right)+C \mu_{k}^{\frac{r}{p^{-}}} .
\end{aligned}
$$

Upon choosing $\epsilon$ appropriately, one obtains

$$
J_{k}(\varrho) \leq C\left(\mu_{k}^{\frac{N r-N-p^{-}}{\left(p^{-}-1\right)\left(N+p^{-}\right)}}+\mu_{k}^{\frac{r}{p^{-}}}\right) .
$$

For any $h>k>0$, it is easy to see

$$
J_{k}(\varrho) \geq\left|A_{h}(\varrho)\right|(h-k)^{r m} .
$$


The relationships (3.23) and (3.24) above imply that

$$
\mu_{h} \leq\left(\frac{M}{h-k}\right)^{r m}\left(\mu_{k}^{1+\frac{N r-p^{-}\left(N+p^{-}\right)}{\left(p^{-}-1\right)\left(N+p^{-}\right)}}+\mu_{k}^{\frac{r}{p^{-}}}\right) .
$$

Noticing that $\frac{N r-p^{-}\left(N+p^{-}\right)}{\left(p^{-}-1\right)\left(N+p^{-}\right)}>0$ and $\frac{r}{p^{-}}>1$, by the iteration Lemma 3.1, we obtain $\mu_{R}=0$ and thus $\left\|u_{\epsilon \eta}\right\|_{L^{\infty}\left(Q_{T}\right)}<R$, where

$$
R=M 2^{\frac{\Lambda}{\Lambda-1}}\left(\left|Q_{T}\right|^{\frac{N r-p^{-}\left(N+p^{-}\right)}{\left(p^{-}-1\right)\left(N+p^{-}\right)}}+\left|Q_{T}\right|^{\frac{r}{p^{-}}-1}\right)^{\frac{1}{r m}}
$$

with

$$
\Lambda=\min \left\{\frac{N r-N-p^{-}}{\left(p^{-}-1\right)\left(N+p^{-}\right)}, \frac{r}{p^{-}}\right\} .
$$

Theorem 3.3 Assume $K \geq 0$, for a.e. $(x, t) \in Q_{T}$. Then there exists a positive constant $R$ such that

$$
\operatorname{deg}\left(u-T_{\epsilon \eta}\left(1, u^{+}\right), B_{R}, 0\right)=1,
$$

where $u^{+}=\max \{u, 0\}$.

Proof From Proposition 3.2, we take $K_{1}=\|a\|_{L^{\infty}\left(Q_{T}\right)}$, it implies that there exists a positive constant $R>0$ independent of $\epsilon$ and $\eta$, such that $u_{\epsilon \eta} \neq G_{\epsilon \eta}\left(1, \rho f\left(u_{\epsilon \eta}^{+}\right)\right)$, for any $u_{\epsilon \eta} \in \partial B_{R}$, $\rho \in[0,1]$. Hence the topological degree $\operatorname{deg}\left(u-G_{\epsilon \eta}\left(1, \rho f\left(u^{+}\right)\right), B_{R}, 0\right)$ is well defined in $B_{R}$. Thanks to the homotopy invariance property of the Leray-Schauder degree, we have

$$
\operatorname{deg}\left(u-G_{\epsilon \eta}\left(1, f\left(u^{+}\right)\right), B_{R}, 0\right)=\operatorname{deg}\left(u-G_{\epsilon \eta}(1,0), B_{R}, 0\right) .
$$

Using the fact that $G_{\epsilon \eta}(1,0)=0$, one has

$$
\operatorname{deg}\left(u-G_{\epsilon \eta}(1,0), B_{R}, 0\right)=\operatorname{deg}\left(I, B_{R}, 0\right)=1 .
$$

From (3.26) and (3.27), we get $\operatorname{deg}\left(u-T_{\epsilon \eta}\left(1, u^{+}\right), B_{R}, 0\right)=1$.

Using the standard method, similar to that in [3] or [13], one can prove the following.

Proposition 3.4 Assume that $a \in L^{\infty}\left(Q_{T}\right), K \in L^{\infty}\left(Q_{T}\right)$. If $u_{\epsilon \eta}$ solves $u=G_{\epsilon \eta}\left(\sigma, \rho f\left(u^{+}\right)+\right.$ $1-\sigma)$, for some $\sigma \in[0,1]$ and $\rho \in[0,1]$, then $u_{\epsilon \eta} \geq 0$ for any $(x, t) \in Q_{T}$. Moreover, if $u_{\epsilon \eta} \neq 0$, then $u_{\epsilon \eta}>0$ in $Q_{T}$.

In what follows, we prove a lower bound for the regularized problem.

Proposition 3.5 Let $\mu_{1}$ be the first eigenvalue of

$$
\begin{cases}-\Delta u=\mu u, & x \in \Omega, \\ u=0, & x \in \partial \Omega,\end{cases}
$$


and let $e_{1}$ be the associated positive eigenfunction such that $\left\|e_{1}\right\|_{L^{2}(\Omega)}=1$. Assume that $\frac{1}{T} \iint_{Q_{T}} a e_{1}^{2} d x d t>\mu_{1}, 0<\epsilon<\frac{1}{2}$ and $0<\eta<\left(\frac{1}{2}\right)^{\frac{p^{+}-2}{p^{-2}}}$. If $u_{\epsilon \eta} \neq 0$ satisfies $u_{\epsilon \eta}=G_{\epsilon \eta}\left(\sigma, f\left(u_{\epsilon \eta}^{+}\right)+\right.$ $1-\sigma)$ for some $\sigma \in[0,1]$, then $\left\|u_{\epsilon \eta}\right\|_{L^{\infty}\left(Q_{T}\right)} \geq r_{0}$, where

$$
\begin{aligned}
& r_{0}=\min \left\{\left(\frac{1}{2 m}\right)^{\frac{1}{m-1}},\left(\frac{\iint_{Q_{T}} a e_{1}^{2} d x d t-\mu_{1} T}{M}\right)^{\gamma_{p}^{+}}\right\}, \\
& \gamma_{p}(x)=\frac{p(x)}{p(x)-2}, \\
& \gamma_{p}^{+}=\max _{\bar{\Omega}} \gamma_{p}(x)=\frac{p^{-}}{p^{-}-2}, \quad \gamma_{p}^{-}=\min _{\bar{\Omega}} \gamma_{p}(x)=\frac{p^{+}}{p^{+}-2}, \\
& M=\|K\|_{L^{1}\left(Q_{T}\right)}+2^{p^{+}-2}\left(\frac{1}{\gamma_{p}^{-}}+\frac{2}{p^{-}}\right) C_{p^{+}, p(x)} \max _{\bar{\Omega}}\left|\nabla e_{1}\right|^{2}|\Omega|^{\frac{2}{p^{+}}} \\
& \quad \times \max \left\{\left(\|a\|_{L^{1}\left(Q_{T}\right)}+|\Omega| T\right)^{\frac{1}{\gamma_{p}^{ \pm}}} T^{\frac{\gamma_{p}^{ \pm}-1}{\gamma_{p}^{ \pm}}}\right\},
\end{aligned}
$$

$C_{p^{+}, p(x)}$ is the embedding constant of $L^{p^{+}}(\Omega)$ into $L^{p(x)}(\Omega)$, and $|\Omega|$ is the Lebesgue measure of the domain $\Omega$.

Proof We argue by contradiction. If not, then for each $\sigma \in[0,1]$ and $r \in\left(0, r_{0}\right)$, there exists a $u_{\epsilon \eta} \neq 0$ such that $u_{\varepsilon \eta}=G_{\epsilon \eta}\left(\sigma, f\left(u_{\varepsilon \eta}^{+}\right)+1-\sigma\right)$, with $\left\|u_{\epsilon \eta}\right\|_{L^{\infty}\left(Q_{T}\right)} \leq r$. For clarity, we divide the proof into four steps.

Step 1 . Note that, by Proposition 3.4, $u_{\epsilon \eta}>0$ in $Q_{T}$. Taking $\phi \in C_{0}^{\infty}(\Omega)$ and multiplying

$$
\begin{gathered}
\frac{\partial u_{\epsilon \eta}}{\partial t}-\operatorname{div}\left\{\left(\left|\nabla\left(\sigma u_{\epsilon \eta}^{m}+\epsilon u_{\epsilon \eta}\right)\right|^{2}+\eta\right)^{\frac{p(x)-2}{2}} \nabla\left(\sigma u_{\epsilon \eta}^{m}+\epsilon u_{\epsilon \eta}\right)\right\} \\
=\left[a-\int_{\Omega} K(\xi, t) u_{\epsilon \eta}^{2}(\xi, t-\tau) d \xi\right] u_{\epsilon \eta}+1-\sigma
\end{gathered}
$$

by $\frac{\phi^{2}}{u_{\epsilon}}$, integrating over $Q_{T}$ and using the $T$-periodicity of $u_{\epsilon \eta}$, we obtain

$$
\begin{aligned}
& \iint_{Q_{T}}\left[a-\int_{\Omega} K(\xi, t) u_{\epsilon \eta}^{2}(\xi, t-\tau) d \xi\right] \phi^{2} d x d t+\iint_{Q_{T}}(1-\sigma) \frac{\phi^{2}}{u_{\epsilon \eta}} d x d t \\
& \quad=-\iint_{Q_{T}} \frac{\phi^{2}}{u_{\epsilon \eta}} \operatorname{div}\left\{\left(\left|\nabla\left(\sigma u_{\epsilon \eta}^{m}+\epsilon u_{\epsilon \eta}\right)\right|^{2}+\eta\right)^{\frac{p(x)-2}{2}} \nabla\left(\sigma u_{\epsilon \eta}^{m}+\epsilon u_{\epsilon \eta}\right)\right\} d x d t \\
& \quad:=(R) .
\end{aligned}
$$

Step 2. Using $\nabla u_{\epsilon \eta} \nabla\left(\frac{\phi^{2}}{u_{\epsilon \eta}}\right)=|\nabla \phi|^{2}-u_{\epsilon \eta}^{2}\left|\nabla\left(\frac{\phi}{u_{\epsilon \eta}}\right)\right|^{2}$, we have

$$
\begin{aligned}
(R) & =\iint_{Q_{T}}\left(\left|\nabla\left(\sigma u_{\epsilon \eta}^{m}+\epsilon u_{\epsilon \eta}\right)\right|^{2}+\eta\right)^{\frac{p(x)-2}{2}} \nabla\left(\sigma u_{\epsilon \eta}^{m}+\epsilon u_{\epsilon \eta}\right) \nabla\left(\frac{\phi^{2}}{u_{\epsilon \eta}}\right) d x d t \\
& =\iint_{Q_{T}}\left(\left|\nabla\left(\sigma u_{\epsilon \eta}^{m}+\epsilon u_{\epsilon \eta}\right)\right|^{2}+\eta\right)^{\frac{p(x)-2}{2}}\left(\sigma m u_{\epsilon \eta}^{m-1}+\epsilon\right) \nabla u_{\epsilon \eta} \nabla\left(\frac{\phi^{2}}{u_{\epsilon \eta}}\right) d x d t \\
& =\iint_{Q_{T}}\left(\left|\nabla\left(\sigma u_{\epsilon \eta}^{m}+\epsilon u_{\epsilon \eta}\right)\right|^{2}+\eta\right)^{\frac{p(x)-2}{2}}\left(\sigma m u_{\epsilon \eta}^{m-1}+\epsilon\right)|\nabla \phi|^{2} d x d t
\end{aligned}
$$


Li and Gao Boundary Value Problems 2014, 2014:77

Page 12 of 21

http://www.boundaryvalueproblems.com/content/2014/1/77

$$
\begin{aligned}
& -\iint_{Q_{T}}\left(\left|\nabla\left(\sigma u_{\epsilon \eta}^{m}+\epsilon u_{\epsilon \eta}\right)\right|^{2}+\eta\right)^{\frac{p(x)-2}{2}}\left(\sigma m u_{\epsilon \eta}^{m-1}+\epsilon\right) u_{\epsilon \eta}^{2}\left|\nabla\left(\frac{\phi}{u_{\epsilon \eta}}\right)\right|^{2} d x d t \\
\leq & \iint_{Q_{T}}\left(\left|\nabla\left(\sigma u_{\epsilon \eta}^{m}+\epsilon u_{\epsilon \eta}\right)\right|^{2}+\eta\right)^{\frac{p(x)-2}{2}}\left(\sigma m u_{\epsilon \eta}^{m-1}+\epsilon\right)|\nabla \phi|^{2} d x d t .
\end{aligned}
$$

Since $r<r_{0} \leq\left(\frac{1}{2 m}\right)^{\frac{1}{m-1}}$ and $0<\epsilon<\frac{1}{2}$, we have $\sigma m u_{\epsilon \eta}^{m-1}+\epsilon \leq m u_{\epsilon \eta}^{m-1}+\epsilon<\frac{1}{2}+\epsilon<1$. Hence

$$
\begin{aligned}
(R) \leq & \iint_{Q_{T}}\left(\left|\nabla\left(\sigma u_{\epsilon \eta}^{m}+\epsilon u_{\epsilon \eta}\right)\right|^{2}+\eta\right)^{\frac{p(x)-2}{2}}|\nabla \phi|^{2} d x d t \\
\leq & 2^{\frac{p^{+}-2}{2}} \iint_{Q_{T}}\left(\left|\nabla\left(\sigma u_{\epsilon \eta}^{m}+\epsilon u_{\epsilon \eta}\right)\right|^{p(x)-2}+\eta^{\frac{p^{-}-2}{2}}\right)|\nabla \phi|^{2} d x d t \\
= & 2^{\frac{p^{+}-2}{2}} \iint_{Q_{T}}\left|\nabla\left(\sigma u_{\epsilon \eta}^{m}+\epsilon u_{\epsilon \eta}\right)\right|^{p(x)-2}|\nabla \phi|^{2} d x d t \\
& +2^{\frac{p^{+}-2}{2}} \eta^{\frac{p^{-}-2}{2}} \iint_{Q_{T}}|\nabla \phi|^{2} d x d t .
\end{aligned}
$$

Thanks to the $p(x)$-Hölder's inequality in variable exponent space, we have

$$
\begin{aligned}
\int_{\Omega} \mid & \left.\nabla\left(\sigma u_{\epsilon \eta}^{m}+\epsilon u_{\epsilon \eta}\right)\right|^{p(x)-2}|\nabla \phi|^{2} d x \\
\leq & \left.\left.\left.\left.\left(\frac{1}{\gamma_{p}^{-}}+\frac{2}{p^{-}}\right)|| \nabla\left(\sigma u_{\epsilon \eta}^{m}+\epsilon u_{\epsilon \eta}\right)\right|^{p(x)-2}\right|_{L^{\gamma p(x)}(\Omega)}|| \nabla \phi\right|^{2}\right|_{L^{\frac{p(x)}{2}}(\Omega)} \\
\leq & \left(\frac{1}{\gamma_{p}^{-}}+\frac{2}{p^{-}}\right) C_{p^{+}, p(x)}\left\||\nabla \phi|^{2}\right\|_{L^{\frac{p^{+}}{2}(\Omega)}} \\
& \times \max \left\{\left(\int_{\Omega}\left|\nabla\left(\sigma u_{\epsilon \eta}^{m}+\epsilon u_{\epsilon \eta}\right)\right|^{p(x)} d x\right)^{\frac{1}{\gamma_{p}^{ \pm}}}\right\} .
\end{aligned}
$$

Noting that $\frac{1}{\gamma_{p}^{ \pm}}<1$ and using Hölder's inequality, we have

$$
\begin{aligned}
& \int_{0}^{T}\left(\int_{\Omega}\left|\nabla\left(\sigma u_{\epsilon \eta}^{m}+\epsilon u_{\epsilon \eta}\right)\right|^{p(x)} d x\right)^{\frac{1}{\gamma_{p}^{ \pm}}} d t \\
& \quad \leq T^{1-\frac{1}{\gamma_{p}^{ \pm}}}\left(\iint_{Q_{T}}\left|\nabla\left(\sigma u_{\epsilon \eta}^{m}+\epsilon u_{\epsilon \eta}\right)\right|^{p(x)} d x d t\right)^{\frac{1}{\gamma_{p}^{ \pm}}} .
\end{aligned}
$$

Integrating (3.31) over $[0, T]$ and noting (3.32), we get

$$
\begin{aligned}
& \iint_{Q_{T}}\left|\nabla\left(\sigma u_{\epsilon \eta}^{m}+\epsilon u_{\epsilon \eta}\right)\right|^{p(x)-2}|\nabla \phi|^{2} d x d t \\
& \leq\left(\frac{1}{\gamma_{p}^{-}}+\frac{2}{p^{-}}\right) C_{p^{+}, p(x)}\left\||\nabla \phi|^{2}\right\|_{L^{\frac{p^{+}}{2}}(\Omega)} \\
& \quad \times \max \left\{\left(\iint_{Q_{T}}\left|\nabla\left(\sigma u_{\epsilon \eta}^{m}+\epsilon u_{\epsilon \eta}\right)\right|^{p(x)} d x d t\right)^{\frac{1}{\gamma_{p}^{ \pm}}} T^{\frac{\gamma_{p}^{ \pm}-1}{\gamma_{p}^{ \pm}}}\right\} .
\end{aligned}
$$


Step 3. Multiplying (3.28) by $\sigma u_{\epsilon \eta}^{m}+\epsilon u_{\epsilon \eta}$, integrating over $Q_{T}$, noticing the $T$-periodicity of $u_{\epsilon \eta}$ and $0<r<r_{0} \leq\left(\frac{1}{2 m}\right)^{\frac{1}{m-1}}<1$, we deduce

$$
\begin{aligned}
& \iint_{Q_{T}}\left|\nabla\left(\sigma u_{\epsilon \eta}^{m}+\epsilon u_{\epsilon \eta}\right)\right|^{p(x)} d x d t \\
& \quad \leq \iint_{Q_{T}}\left(\left|\nabla\left(\sigma u_{\epsilon \eta}^{m}+\epsilon u_{\epsilon \eta}\right)\right|^{2}+\eta\right)^{\frac{p(x)-2}{2}}\left|\nabla\left(\sigma u_{\epsilon \eta}^{m}+\epsilon u_{\epsilon \eta}\right)\right|^{2} d x d t \\
& \quad \leq \iint_{Q_{T}} a u_{\epsilon \eta}\left(u_{\epsilon \eta}^{m}+u_{\epsilon \eta}\right) d x d t+(1-\sigma) \iint_{Q_{T}}\left(u_{\epsilon \eta}^{m}+u_{\epsilon \eta}\right) d x d t \\
& \quad \leq\left(\left\|u_{\epsilon \eta}\right\|_{L^{\infty}\left(Q_{T}\right)}^{m+1}+\left\|u_{\epsilon \eta}\right\|_{L^{\infty}\left(Q_{T}\right)}^{2}\right)\|a\|_{L^{1}\left(Q_{T}\right)} \\
& \quad+(1-\sigma)\left(\left\|u_{\epsilon \eta}\right\|_{L^{\infty}\left(Q_{T}\right)}^{m}+\left\|u_{\epsilon \eta}\right\|_{L^{\infty}\left(Q_{T}\right)}\right)|\Omega| T \\
& \quad \leq\left(r^{m+1}+r^{2}\right)\|a\|_{L^{1}\left(Q_{T}\right)}+\left(r^{m}+r\right)|\Omega| T \\
& \leq 2 r\left(\|a\|_{L^{1}\left(Q_{T}\right)}+|\Omega| T\right) .
\end{aligned}
$$

Substituting this inequality into (3.33), we have

$$
\begin{aligned}
& \iint_{Q_{T}}\left|\nabla\left(\sigma u_{\epsilon \eta}^{m}+\epsilon u_{\epsilon \eta}\right)\right|^{p(x)-2}|\nabla \phi|^{2} d x d t \\
& \leq\left(\frac{1}{\gamma_{p}^{-}}+\frac{2}{p^{-}}\right) C_{p^{+}, p(x)}\left\||\nabla \phi|^{2}\right\|_{L^{\frac{p^{+}}{2}}(\Omega)} \\
& \quad \times \max \left\{\left(2 r\left(\|a\|_{L^{1}\left(Q_{T}\right)}+|\Omega| T\right)\right)^{\frac{1}{\gamma_{p}^{ \pm}}} T^{\frac{\gamma_{p}^{ \pm}-1}{\gamma_{p}^{ \pm}}}\right\} \\
& \leq\left(\frac{1}{\gamma_{p}^{-}}+\frac{2}{p^{-}}\right) C_{p^{+}, p(x)}\left\||\nabla \phi|^{2}\right\|_{L^{\frac{p^{+}}{2}}(\Omega)} 2^{\frac{1}{\gamma_{p}^{p}}} r^{\frac{1}{\gamma_{p}^{+}}} \\
& \quad \times \max \left\{\left(\|a\|_{L^{1}\left(Q_{T}\right)}+|\Omega| T\right)^{\frac{1}{\gamma_{p}^{ \pm}}} T^{\frac{\gamma_{p}^{ \pm}-1}{\gamma_{p}^{ \pm}}}\right\} .
\end{aligned}
$$

Substituting (3.34) into (3.30) and noticing that $2^{\frac{p^{+}-2}{2}} 2^{\frac{1}{\gamma_{p}}} \leq 2^{p^{+}-2}$, we get

$$
\begin{aligned}
(R) \leq & 2^{p^{+}-2}\left(\frac{1}{\gamma_{p}^{-}}+\frac{2}{p^{-}}\right) r^{\frac{1}{\gamma_{p}^{+}}} C_{p^{+}, p(x)}\left\||\nabla \phi|^{2}\right\|_{L^{\frac{p^{+}}{2}}(\Omega)} \\
& \times \max \left\{\left(\|a\|_{L^{1}\left(Q_{T}\right)}+|\Omega| T\right)^{\frac{1}{\gamma_{p}^{ \pm}}} T^{\frac{\gamma_{p}^{ \pm}-1}{\gamma_{p}^{ \pm}}}\right\}+2^{\frac{p^{+}-2}{2}} \eta^{\frac{p^{-}-2}{2}} \iint_{Q_{T}}|\nabla \phi|^{2} d x d t .
\end{aligned}
$$

Considering that $0<\eta<\left(\frac{1}{2}\right)^{\frac{p^{+}-2}{p^{-}-2}}$, from (3.29) and (3.35), we have

$$
\begin{aligned}
& \iint_{Q_{T}}\left[a-\int_{\Omega} K(\xi, t) u_{\epsilon \eta}^{2}(\xi, t-\tau) d \xi\right] \phi^{2} d x d t \\
& \leq \iint_{Q_{T}}|\nabla \phi|^{2} d x d t+2^{p^{+}-2}\left(\frac{1}{\gamma_{p}^{-}}+\frac{2}{p^{-}}\right) r^{\frac{1}{\gamma_{p}^{+}}} C_{p^{+}, p(x)}\left\||\nabla \phi|^{2}\right\|_{L^{\frac{p^{+}}{2}}(\Omega)} \\
& \quad \times \max \left\{\left(\|a\|_{L^{1}\left(Q_{T}\right)}+|\Omega| T\right)^{\frac{1}{\gamma_{p}^{ \pm}}} T^{\frac{\gamma_{p}^{ \pm}-1}{\gamma_{p}^{ \pm}}}\right\} .
\end{aligned}
$$


Step 4 . We claim

$$
\iint_{Q_{T}} a e_{1}^{2} d x d t-\mu_{1} T \leq r^{\frac{1}{\gamma_{p}^{+}}} M
$$

from which we will derive a contradiction. First, to show (3.37), let $\phi=e_{1}$ in (3.36). Using the fact that $\nabla e_{1} \in\left(C^{1}(\bar{\Omega})\right)^{N}$ and noting $\left\|e_{1}\right\|_{L^{2}(\Omega)}=1$ and $\int_{\Omega}\left|\nabla e_{1}\right|^{2} d x=\mu_{1}$, we get

$$
\begin{aligned}
& \iint_{Q_{T}} a e_{1}^{2} d x d t-\mu_{1} T \\
& \leq 2^{p^{+}-2}\left(\frac{1}{\gamma_{p}^{-}}+\frac{2}{p^{-}}\right) r^{\frac{1}{\gamma_{p}^{+}}} C_{p^{+}, p(x)}\left\|\left|\nabla e_{1}\right|^{2}\right\|_{L^{\frac{p^{+}}{2}}(\Omega)} \\
& \quad \times \max \left\{\left(\|a\|_{L^{1}\left(Q_{T}\right)}+|\Omega| T\right)^{\frac{1}{\gamma_{p}^{ \pm}}} T^{\frac{\gamma_{p}^{ \pm}-1}{\gamma_{p}^{ \pm}}}\right\} \\
& \quad+\int_{0}^{T} \int_{\Omega} K(\xi, t) u_{\epsilon \eta}^{2}(\xi, t-\tau) d \xi \int_{\Omega} e_{1}^{2} d x d t \\
& \leq 2^{p^{+}-2}\left(\frac{1}{\gamma_{p}^{-}}+\frac{2}{p^{-}}\right) r^{\frac{1}{\gamma_{p}^{+}}} C_{p^{+}, p(x)} \max _{\bar{\Omega}}\left|\nabla e_{1}\right|^{2}|\Omega|^{\frac{2}{p^{+}}} \\
& \quad \times \max \left\{\left(\|a\|_{L^{1}\left(Q_{T}\right)}+|\Omega| T\right)^{\frac{1}{\gamma_{p}^{ \pm}}} T_{\frac{\gamma_{p}^{ \pm}-1}{\gamma_{p}^{ \pm}}}^{\gamma^{ \pm}}\right\}+\|K\|_{L^{1}\left(Q_{T}\right)^{2}} r^{2} \\
& \leq r^{\frac{1}{\gamma_{p}^{+}}}\left[2^{p^{+}-2}\left(\frac{1}{\gamma_{p}^{-}}+\frac{2}{p^{-}}\right) C_{p^{+}, p(x)} \max _{\bar{\Omega}}\left|\nabla e_{1}\right|^{2}|\Omega|^{\frac{2}{p^{+}}}\right. \\
&\left.\quad \times \max \left\{\left(\|a\|_{L^{1}\left(Q_{T}\right)}+|\Omega| T\right)^{\frac{1}{\gamma_{p}^{ \pm}}} T_{\frac{\gamma_{p}^{ \pm}-1}{\gamma_{p}^{ \pm}}}^{\gamma^{ \pm}}\right\}+\|K\|_{L^{1}\left(Q_{T}\right)}\right] \\
&= r^{\frac{1}{\gamma_{p}^{+}}} M .
\end{aligned}
$$

Now the definition of $r_{0}$ and (3.37) yield

$$
r_{0} \leq\left(\frac{\iint_{Q_{T}} a e_{1}^{2} d x d t-\mu_{1} T}{M}\right)^{\gamma_{p}^{+}} \leq r
$$

which is clearly a contradiction to the assumption that $r \in\left(0, r_{0}\right)$. This completes the proof.

Theorem 3.6 Let $r_{0}$ be as given in Proposition 3.5. Then $\operatorname{deg}\left(u-T_{\epsilon \eta}\left(1, u^{+}\right), B_{r}, 0\right)=0$ for all $0<r<r_{0}$.

Proof In view of Proposition 3.5, for any fixed $r \in\left(0, r_{0}\right)$, we have proved that $u \neq$ $G_{\epsilon \eta}\left(\sigma, f\left(u^{+}\right)+1-\sigma\right)$ for all $u \in \partial B_{r}, \sigma \in[0,1]$. So the Leray-Schauder topological degree $\operatorname{deg}\left(u-G_{\epsilon \eta}\left(\sigma, f\left(u^{+}\right)+1-\sigma\right), B_{r}, 0\right)$ is well defined for all $\sigma \in[0,1]$. Thanks to the homotopy invariance of the topological degree, we have

$$
\operatorname{deg}\left(u-G_{\epsilon \eta}\left(1, f\left(u^{+}\right)\right), B_{r}, 0\right)=\operatorname{deg}\left(u-G_{\epsilon \eta}\left(0, f\left(u^{+}\right)+1\right), B_{r}, 0\right) .
$$


Also, from Proposition 3.5, we infer that $u=G_{\epsilon \eta}\left(0, f\left(u^{+}\right)+1\right)$ admits no nontrivial solution in $B_{r}$. Moreover, $u_{\epsilon \eta}=0$ is not a solution of $u=G_{\epsilon \eta}\left(0, f\left(u^{+}\right)+1\right)$. So $\operatorname{deg}\left(u-G_{\epsilon \eta}\left(0, f\left(u^{+}\right)+\right.\right.$ $\left.1), B_{r}, 0\right)=0$. Together with (3.39), we have $\operatorname{deg}\left(u-T_{\epsilon \eta}\left(1, u^{+}\right), B_{r}, 0\right)=0$.

\section{Existence of nontrivial nonnegative solution to (1.1)}

Theorem 4.1 Assume $K(x, t) \geq 0$ for a.e. $(x, t) \in Q_{T}$ and $\frac{1}{T} \iint_{Q_{T}} a e_{1}^{2} d x d t>\mu_{1}$. Then problem (1.1) has a nontrivial nonnegative periodic solution.

Proof We consider the regularized problem (2.2). By Theorem 3.3 and Theorem 3.6, we conclude that there exist $R$ and $r$, independent of $\epsilon$ and $\eta$, with $R>r>0$, such that

$$
\operatorname{deg}\left(u-G_{\epsilon \eta}\left(1, f\left(u^{+}\right)\right), B_{R} \backslash \bar{B}_{r}, 0\right)=1
$$

for $0<\epsilon<\frac{1}{2}$ and $0<\eta<\left(\frac{1}{2}\right)^{\frac{p^{+}-2}{p--2}}$. Using the solvability of the Leray-Schauder degree, we conclude that the regularized problem (2.2) admits a nontrivial nonnegative solution $u_{\epsilon \eta}$ in $B_{R} \backslash \bar{B}_{r}$.

We prove that $u_{\epsilon \eta}^{m} \in L^{p^{-}}\left(0, T ; W_{0}^{1, p(x)}(\Omega)\right)$ with $\left|\nabla u_{\epsilon \eta}^{m}\right| \in L^{p(x)}\left(Q_{T}\right)$ and that a solution to problem (1.1) is obtained as a limit of $u_{\epsilon \eta}$ as $\epsilon, \eta \rightarrow 0$. We proceed in several steps.

Step 1 . In view of $K(x, t) \geq 0$, choosing $C=\|a\|_{L^{\infty}\left(Q_{T}\right)}$, we have

$$
\frac{\partial u_{\epsilon \eta}}{\partial t}-\operatorname{div}\left\{\left(\left|\nabla\left(u_{\epsilon \eta}^{m}+\epsilon u_{\epsilon \eta}\right)\right|^{2}+\eta\right)^{\frac{p(x)-2}{2}} \nabla\left(u_{\epsilon \eta}^{m}+\epsilon u_{\epsilon \eta}\right)\right\} \leq C u_{\epsilon \eta} .
$$

Multiplying (4.1) by $u_{\epsilon \eta}^{m}+\epsilon u_{\epsilon \eta}$, integrating over $Q_{T}$ and noting the $T$-periodicity of $u_{\epsilon \eta}$ and the boundedness of $u_{\epsilon \eta}$, we have

$$
\begin{aligned}
& \iint_{Q_{T}}\left|\nabla u_{\epsilon \eta}^{m}\right|^{p(x)} d x d t \\
& \quad \leq \iint_{Q_{T}}\left(\left|\nabla\left(u_{\epsilon \eta}^{m}+\epsilon u_{\epsilon \eta}\right)\right|^{2}+\eta\right)^{\frac{p(x)-2}{2}}\left|\nabla\left(u_{\epsilon \eta}^{m}+\epsilon u_{\epsilon \eta}\right)\right|^{2} d x d t \\
& \quad \leq C \iint_{Q_{T}}\left(u_{\epsilon \eta}^{m+1}+\epsilon u_{\epsilon \eta}^{2}\right) d x d t \leq M,
\end{aligned}
$$

where $M$ is a positive constant independent of $\epsilon$ and $\eta$. Moreover,

$$
\begin{aligned}
& \int_{0}^{T}\left|\nabla u_{\epsilon \eta}^{m}\right|_{L^{p(x)}(\Omega)}^{p^{-}} d t \\
& =\int_{[0, T] \cap\left\{t:\left|\nabla u_{\epsilon \eta}^{m}\right|_{L^{p(x)}(\Omega)}>1\right\}}\left|\nabla u_{\epsilon \eta}^{m}\right|_{L^{p(x)}(\Omega)}^{p^{-}} d t \\
& \quad+\int_{[0, T] \cap\left\{t:\left|\nabla u_{\epsilon \eta}^{m}\right|_{L^{p(x)}(\Omega)} \leq 1\right\}}\left|\nabla u_{\epsilon \eta}^{m}\right|_{L^{p(x)}(\Omega)}^{p^{-}} d t \\
& \leq \int_{[0, T] \cap\left\{t:\left|\nabla u_{\epsilon \eta}^{m}\right|_{L^{p(x)}(\Omega)}>1\right\}} \int_{\Omega}\left|\nabla u_{\epsilon \eta}^{m}\right|^{p(x)} d x d t+T \\
& \leq \iint_{Q_{T}}\left|\nabla u_{\epsilon \eta}^{m}\right|^{p(x)} d x d t+T \leq M+T .
\end{aligned}
$$


So $u_{\epsilon \eta}^{m} \in L^{p^{-}}\left(0, T ; W_{0}^{1, p(x)}(\Omega)\right)$ and $u_{\epsilon \eta}^{m}$ is uniformly bounded in the space $L^{p^{-}}(0, T$; $\left.W_{0}^{1, p(x)}(\Omega)\right)$. Thus, up to subsequence if necessary, we may assume that $u_{\epsilon \eta}^{m} \rightarrow u^{m} \in$ $L^{p^{-}}\left(0, T ; W_{0}^{1, p(x)}(\Omega)\right)$. In what follows, our main goal is to prove that $u$ is a weak solution of problem (1.1).

Step 2. The following relation is obvious:

$$
\begin{aligned}
& \iint_{Q_{T}}\left(\left|\nabla\left(u_{\epsilon \eta}^{m}+\epsilon u_{\epsilon \eta}\right)\right|^{2}+\eta\right)^{\frac{p(x)-2}{2}}\left|\nabla\left(u_{\epsilon \eta}^{m}+\epsilon u_{\epsilon \eta}\right)\right|^{2} d x d t \\
& \quad \geq \iint_{Q_{T}}\left|\nabla\left(u_{\epsilon \eta}^{m}+\epsilon u_{\epsilon \eta}\right)\right|^{p(x)} d x d t .
\end{aligned}
$$

From (4.2) and (4.4), we have

$$
\iint_{Q_{T}}\left|\nabla\left(u_{\epsilon \eta}^{m}+\epsilon u_{\epsilon \eta}\right)\right|^{p(x)} d x d t \leq C
$$

Owing to the embedding results in the variable exponent space, one has

$$
\begin{gathered}
\left\|\nabla\left(u_{\epsilon \eta}^{m}+\epsilon u_{\epsilon \eta}\right)\right\|_{L^{2}(\Omega)} \leq C_{2, p(x)}\left|\nabla\left(u_{\epsilon \eta}^{m}+\epsilon u_{\epsilon \eta}\right)\right|_{L^{p(x)}(\Omega)} \\
\leq C_{2, p(x)} \max \left\{\left(\int_{\Omega}\left|\nabla\left(u_{\epsilon \eta}^{m}+\epsilon u_{\epsilon \eta}\right)\right|^{p(x)} d x\right)^{\frac{1}{p^{ \pm}}}\right\} .
\end{gathered}
$$

Integrating (4.6) over $[0, T]$ and using Hölder's inequality, we have

$$
\begin{aligned}
& \int_{0}^{T}\left\|\nabla\left(u_{\epsilon \eta}^{m}+\epsilon u_{\epsilon \eta}\right)\right\|_{L^{2}(\Omega)} d t \\
& \quad \leq C_{2, p(x)} \max \left\{\left(\iint_{Q_{T}}\left|\nabla\left(u_{\epsilon \eta}^{m}+\epsilon u_{\epsilon \eta}\right)\right|^{p(x)} d x d t\right)^{\frac{1}{p^{ \pm}}} T^{1-\frac{1}{p^{ \pm}}}\right\} .
\end{aligned}
$$

From (4.5) and (4.7), there exists a positive constant $C$ independent of $\epsilon$ and $\eta$, such that

$$
\iint_{Q_{T}}\left|\nabla\left(u_{\epsilon \eta}^{m}+\epsilon u_{\epsilon \eta}\right)\right|^{2} d x d t \leq C
$$

In the following, we prove

$$
\iint_{Q_{T}}\left|\left(\left|\nabla\left(u_{\epsilon \eta}^{m}+\epsilon u_{\epsilon \eta}\right)\right|^{2}+\eta\right)^{\frac{p(x)-2}{2}} \nabla\left(u_{\epsilon \eta}^{m}+\epsilon u_{\epsilon \eta}\right)\right|^{\frac{p(x)}{p(x)-1}} d x d t \leq C .
$$

First, denote

$$
\begin{array}{lll}
K_{1 p}(x)=\frac{p(x)}{p(x)-1}, & K_{1 p}^{-}=\min _{\bar{\Omega}} K_{1 p}(x), & K_{1 p}^{+}=\max _{\bar{\Omega}} K_{1 p}(x), \\
K_{2 p}(x)=\frac{2(p(x)-1)}{p(x)}, & K_{2 p}^{-}=\min _{\bar{\Omega}} K_{2 p}(x), & K_{2 p}^{+}=\max _{\bar{\Omega}} K_{2 p}(x), \\
K_{2 p}^{\prime}(x)=\frac{2(p(x)-1)}{p(x)-2}, & K_{2 p}^{\prime-}=\min _{\bar{\Omega}} K_{2 p}^{\prime}(x), & K_{2 p}^{\prime+}=\max _{\bar{\Omega}} K_{2 p}^{\prime}(x) .
\end{array}
$$


A straightforward computation shows that

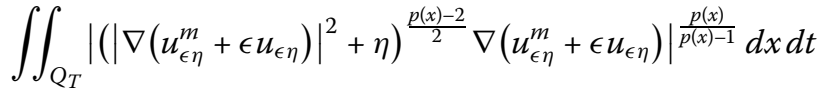

$$
\begin{aligned}
& \leq \iint_{Q_{T}}\left|2^{\frac{p^{+}-2}{2}}\left(\left|\nabla\left(u_{\epsilon \eta}^{m}+\epsilon u_{\epsilon \eta}\right)\right|^{p(x)-2}+\eta^{\frac{p^{-}-2}{2}}\right) \nabla\left(u_{\epsilon \eta}^{m}+\epsilon u_{\epsilon \eta}\right)\right|^{\frac{p(x)}{p(x)-1}} d x d t \\
& \leq 2^{\frac{p^{+}-2}{2} K_{1 p}^{+}} \iint_{Q_{T}} \|\left.\nabla\left(u_{\epsilon \eta}^{m}+\epsilon u_{\epsilon \eta}\right)\right|^{p(x)-2} \nabla\left(u_{\epsilon \eta}^{m}+\epsilon u_{\epsilon \eta}\right)+\left.\nabla\left(u_{\epsilon \eta}^{m}+\epsilon u_{\epsilon \eta}\right)\right|^{\frac{p(x)}{p(x)-1}} d x d t \\
& \leq 2^{\frac{p^{+}-2}{2} K_{1 p}^{+}} 2^{K_{1 p}^{+}} \iint_{Q_{T}}\left(\left|\nabla\left(u_{\epsilon \eta}^{m}+\epsilon u_{\epsilon \eta}\right)\right|^{p(x)}+\left|\nabla\left(u_{\epsilon \eta}^{m}+\epsilon u_{\epsilon \eta}\right)\right|^{\frac{p(x)}{p(x)-1}}\right) d x d t \\
& \leq M_{p}\left\{\iint_{Q_{T}}\left|\nabla\left(u_{\epsilon \eta}^{m}+\epsilon u_{\epsilon \eta}\right)\right|^{p(x)} d x d t+\iint_{Q_{T}}\left|\nabla\left(u_{\epsilon \eta}^{m}+\epsilon u_{\epsilon \eta}\right)\right|^{\frac{p(x)}{p(x)-1}} d x d t\right\} .
\end{aligned}
$$

By the $p(x)$-Hölder's inequality, we have

$$
\begin{aligned}
& \int_{\Omega} \mid\left.\nabla\left(u_{\epsilon \eta}^{m}+\epsilon u_{\epsilon \eta}\right)\right|^{\frac{p(x)}{p(x)-1}} d x \\
& \leq\left.\left.\left(\frac{1}{K_{2 p}^{-}}+\frac{1}{K_{2 p}^{\prime-}}\right)|| \nabla\left(u_{\epsilon \eta}^{m}+\epsilon u_{\epsilon \eta}\right)\right|^{\frac{p(x)}{p(x)-1}}\right|_{L^{K_{2 p}(x)}(\Omega)}|1|_{L^{K_{2 p}^{\prime}}(x)}(\Omega) \\
& \leq\left(\frac{1}{K_{2 p}^{-}}+\frac{1}{K_{2 p}^{\prime-}}\right) \max \left\{|\Omega|^{\frac{1}{K_{2 p}^{\prime+}}},|\Omega|^{\frac{1}{K_{2 p}^{\prime-}}}\right\} \\
& \quad \times \max \left\{\left(\int_{\Omega}\left|\nabla\left(u_{\epsilon \eta}^{m}+\epsilon u_{\epsilon \eta}\right)\right|^{2} d x\right)^{\frac{1}{K_{2 p}^{ \pm}}}\right\} .
\end{aligned}
$$

Integrating (4.11) over [0,T], using the $p(x)$-Hölder's inequality again, we get

$$
\begin{aligned}
& \iint_{Q_{T}}\left|\nabla\left(u_{\epsilon \eta}^{m}+\epsilon u_{\epsilon \eta}\right)\right|^{\frac{p(x)}{p(x)-1}} d x d t \\
& \leq\left(\frac{1}{K_{2 p}^{-}}+\frac{1}{K_{2 p}^{\prime-}}\right) \max \left\{|\Omega|^{\frac{1}{K_{2 p}^{\prime+}}},|\Omega|^{\frac{1}{K_{2 p}^{\prime}}}\right\} \max \left\{T^{\frac{1}{K_{2 p}^{1+}}}, T^{\frac{1}{K_{2 p}^{\prime-}}}\right\} \\
& \quad \times \max \left\{\left(\iint_{Q_{T}}\left|\nabla\left(u_{\epsilon \eta}^{m}+\epsilon u_{\epsilon \eta}\right)\right|^{2} d x d t\right)^{\frac{1}{K_{2 p}^{ \pm}}}\right\} .
\end{aligned}
$$

Substituting (4.5), (4.8), and (4.12) into (4.10), we derive (4.9). Therefore, there exists a $H \in\left(L^{\frac{p(x)}{p(x)-1}}\left(Q_{T}\right)\right)^{N}$ such that

$$
\left(\left|\nabla\left(u_{\epsilon \eta}^{m}+\epsilon u_{\epsilon \eta}\right)\right|^{2}+\eta\right)^{\frac{p(x)-2}{2}} \nabla\left(u_{\epsilon \eta}^{m}+\epsilon u_{\epsilon \eta}\right) \rightarrow H
$$

weakly in $\left(L^{\frac{p(x)}{p(x)-1}}\left(Q_{T}\right)\right)^{N}$ as $\epsilon, \eta \rightarrow 0$.

Step 3. Using a method analogous to [7], we get $\left\|\frac{\partial u_{\epsilon \eta}^{m}}{\partial t}\right\|_{L^{2}\left(Q_{T}\right)} \leq C$, where $C$ is independent of $\epsilon$ and $\eta$. Since $u_{\epsilon \eta}^{m}$ is uniformly bounded in $L^{p^{-}}\left(0, T ; W_{0}^{1, p(x)}(\Omega)\right)$, and $W_{0}^{1, p(x)}(\Omega) \hookrightarrow$ compact $L^{p(x)}(\Omega) \hookrightarrow L^{1}(\Omega)$, by compactness theorem (Corollary 4 in [16]), it follows that $u_{\epsilon \eta}^{m} \rightarrow u^{m}$ in $L^{p^{-}}\left(0, T ; L^{p(x)}(\Omega)\right)$. Thus, we have

$$
0=\iint_{Q_{T}}\left\{-u \frac{\partial \varphi}{\partial t}+H \nabla \varphi-a u \varphi+u \varphi \int_{\Omega} K(\xi, t) u^{2}(\xi, t-\tau) d \xi\right\} d x d t
$$


for any $\varphi \in C^{1}\left(\bar{Q}_{T}\right)$ satisfying $\varphi(x, T)=\varphi(x, 0)$ for $x \in \Omega$ and $\left.\varphi(\cdot, t)\right|_{\partial \Omega}=0$ for $t \in[0, T]$

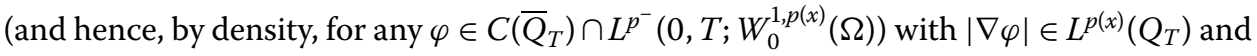
$T$-periodicity). The continuity of $u$ follows from similar Hölder estimates in [17].

Step 4 . It remains to verify for any $\varphi \in C^{1}\left(\bar{Q}_{T}\right)$,

$$
\iint_{Q_{T}}\left|\nabla u^{m}\right|^{p(x)-2} \nabla u^{m} \nabla \varphi d x d t=\iint_{Q_{T}} H \nabla \varphi d x d t
$$

We consider matrix function $\Pi(Y)=\left(|Y|^{2}+\eta\right)^{\frac{p(x)-2}{2}} Y$. Then $\Pi^{\prime}(Y)=\left(|Y|^{2}+\eta\right)^{\frac{p(x)-2}{2}} I+$ $(p(x)-2)\left(|Y|^{2}+\eta\right)^{\frac{p(x)-4}{2}} Y Y^{T}$ is a positive definite matrix. Choosing $v \in L^{p^{-}}\left(0, T ; W_{0}^{1, p(x)}(\Omega)\right)$ with $|\nabla v| \in L^{p(x)}\left(Q_{T}\right)$, by mean value theorem, there exists a matrix $Y$ such that

$$
\begin{aligned}
& \left\langle\Pi\left(\nabla\left(u_{\epsilon \eta}^{m}+\epsilon u_{\epsilon \eta}\right)\right)-\Pi(\nabla v), \nabla\left(u_{\epsilon \eta}^{m}+\epsilon u_{\epsilon \eta}\right)-\nabla v\right\rangle \\
& \quad=\left\langle\Pi^{\prime}(Y)\left(\nabla\left(u_{\epsilon \eta}^{m}+\epsilon u_{\epsilon \eta}\right)-\nabla v\right), \nabla\left(u_{\epsilon \eta}^{m}+\epsilon u_{\epsilon \eta}\right)-\nabla v\right\rangle \geq 0,
\end{aligned}
$$

which gives

$$
\begin{aligned}
0 \leq & \iint_{Q_{T}}\left\{\left(\left|\nabla\left(u_{\epsilon \eta}^{m}+\epsilon u_{\epsilon \eta}\right)\right|^{2}+\eta\right)^{\frac{p(x)-2}{2}} \nabla\left(u_{\epsilon \eta}^{m}+\epsilon u_{\epsilon \eta}\right)\right. \\
& \left.-\left(|\nabla v|^{2}+\eta\right)^{\frac{p(x)-2}{2}} \nabla v\right\} \nabla\left[\left(u_{\epsilon \eta}^{m}+\epsilon u_{\epsilon \eta}\right)-v\right] d x d t \\
= & \iint_{Q_{T}}\left(\left|\nabla\left(u_{\epsilon \eta}^{m}+\epsilon u_{\epsilon \eta}\right)\right|^{2}+\eta\right)^{\frac{p(x)-2}{2}}\left|\nabla\left(u_{\epsilon \eta}^{m}+\epsilon u_{\epsilon \eta}\right)\right|^{2} d x d t \\
& -\iint_{Q_{T}}\left(\left|\nabla\left(u_{\epsilon \eta}^{m}+\epsilon u_{\epsilon \eta}\right)\right|^{2}+\eta\right)^{\frac{p(x)-2}{2}} \nabla\left(u_{\epsilon \eta}^{m}+\epsilon u_{\epsilon \eta}\right) \nabla v d x d t \\
& -\iint_{Q_{T}}\left(|\nabla v|^{2}+\eta\right)^{\frac{p(x)-2}{2}} \nabla \nu \nabla\left[\left(u_{\epsilon \eta}^{m}+\epsilon u_{\epsilon \eta}\right)-v\right] d x d t .
\end{aligned}
$$

Multiplying the equation

$$
\begin{gathered}
\frac{\partial u_{\epsilon \eta}}{\partial t}-\operatorname{div}\left\{\left(\left|\nabla\left(u_{\epsilon \eta}^{m}+\epsilon u_{\epsilon \eta}\right)\right|^{2}+\eta\right)^{\frac{p(x)-2}{2}} \nabla\left(u_{\epsilon \eta}^{m}+\epsilon u_{\epsilon \eta}\right)\right\} \\
=\left[a-\int_{\Omega} K(\xi, t) u_{\epsilon \eta}^{2}(\xi, t-\tau) d \xi\right] u_{\epsilon \eta}
\end{gathered}
$$

by $u_{\epsilon \eta}^{m}+\epsilon u_{\epsilon \eta}$, integrating over $Q_{T}$ and using $T$-periodicity of $u_{\epsilon \eta}$, one has

$$
\begin{aligned}
& \iint_{Q_{T}}\left(\left|\nabla\left(u_{\epsilon \eta}^{m}+\epsilon u_{\epsilon \eta}\right)\right|^{2}+\eta\right)^{\frac{p(x)-2}{2}}\left|\nabla\left(u_{\epsilon \eta}^{m}+\epsilon u_{\epsilon \eta}\right)\right|^{2} d x d t \\
& \quad=\iint_{Q_{T}}\left[a-\int_{\Omega} K(\xi, t) u_{\epsilon \eta}^{2}(\xi, t-\tau) d \xi\right]\left(u_{\epsilon \eta}^{m+1}+\epsilon u_{\epsilon \eta}^{2}\right) d x d t .
\end{aligned}
$$


Thus, (4.17) and (4.18) imply

$$
\begin{aligned}
& \iint_{Q_{T}}\left(\left|\nabla\left(u_{\epsilon \eta}^{m}+\epsilon u_{\epsilon \eta}\right)\right|^{2}+\eta\right)^{\frac{p(x)-2}{2}} \nabla\left(u_{\epsilon \eta}^{m}+\epsilon u_{\epsilon \eta}\right) \nabla v d x d t \\
& \quad+\iint_{Q_{T}}\left(|\nabla v|^{2}+\eta\right)^{\frac{p(x)-2}{2}} \nabla v \nabla\left[\left(u_{\epsilon \eta}^{m}+\epsilon u_{\epsilon \eta}\right)-v\right] d x d t \\
& \quad \leq \iint_{Q_{T}}\left[a-\int_{\Omega} K(\xi, t) u_{\epsilon \eta}^{2}(\xi, t-\tau) d \xi\right]\left(u_{\epsilon \eta}^{m+1}+\epsilon u_{\epsilon \eta}^{2}\right) d x d t
\end{aligned}
$$

Letting $\epsilon, \eta \rightarrow 0$, by (4.13), we have

$$
\begin{gathered}
\iint_{Q_{T}} H \nabla v d x d t+\iint_{Q_{T}}|\nabla v|^{p(x)-2} \nabla v \nabla\left(u^{m}-v\right) d x d t \\
\leq \iint_{Q_{T}}\left[a-\int_{\Omega} K(\xi, t) u^{2}(\xi, t-\tau) d \xi\right] u^{m+1} d x d t .
\end{gathered}
$$

Let $\varphi=u^{m}$ in (4.14) and, by the $T$-periodicity of $u$, we get

$$
\iint_{Q_{T}} H \nabla u^{m} d x d t=\iint_{Q_{T}}\left[a-\int_{\Omega} K(\xi, t) u^{2}(\xi, t-\tau) d \xi\right] u^{m+1} d x d t
$$

Combining (4.19) with (4.20), we obtain

$$
0 \leq \iint_{Q_{T}}\left(H-|\nabla v|^{p(x)-2} \nabla v\right) \nabla\left(u^{m}-v\right) d x d t
$$

Taking $v=u^{m}-\lambda \varphi$, with $\lambda>0$ and $\varphi \in C^{1}\left(\bar{Q}_{T}\right)$, we get

$$
0 \leq \iint_{Q_{T}}\left(H-\left|\nabla\left(u^{m}-\lambda \varphi\right)\right|^{p(x)-2} \nabla\left(u^{m}-\lambda \varphi\right)\right) \nabla \varphi d x d t .
$$

Letting $\lambda \rightarrow 0$ in (4.22) yields

$$
0 \leq \iint_{Q_{T}}\left(H-\left|\nabla u^{m}\right|^{p(x)-2} \nabla u^{m}\right) \nabla \varphi d x d t
$$

On the other hand, if we take $v=u^{m}+\lambda \varphi$, with $\lambda>0$ and $\varphi \in C^{1}\left(\bar{Q}_{T}\right)$ and let $\lambda \rightarrow 0$, we get

$$
0 \geq \iint_{Q_{T}}\left(H-\left|\nabla u^{m}\right|^{p(x)-2} \nabla u^{m}\right) \nabla \varphi d x d t
$$

From (4.23) and (4.24) we have (4.15). This completes the proof of Theorem 4.1.

\section{Appendix}

In this appendix, we prove Lemma 3.1 for the reader's convenience.

Proof of Lemma 3.1 Define the following sequence:

$$
k_{s}=k_{0}+d-\frac{d}{2^{s}}, \quad s=0,1,2, \ldots
$$


where $d$ is to be determined later. Then (3.1) implies the recursive relationship

$$
\varphi\left(k_{s+1}\right) \leq \frac{M^{\alpha} 2^{(s+1) \alpha}}{d^{\alpha}}\left[\varphi^{\beta}\left(k_{s}\right)+\varphi^{\gamma}\left(k_{s}\right)\right], \quad s=0,1,2, \ldots
$$

By induction, one has

$$
\varphi\left(k_{s}\right) \leq \frac{\varphi\left(k_{0}\right)}{r^{s}}, \quad s=0,1,2, \ldots
$$

where $r>1$ is to be chosen. In fact, if (5.2) is right, then

$$
\begin{aligned}
\varphi\left(k_{s+1}\right) & \leq \frac{M^{\alpha} 2^{(s+1) \alpha}}{d^{\alpha}}\left[\frac{\varphi^{\beta}\left(k_{0}\right)}{r^{s \beta}}+\frac{\varphi^{\gamma}\left(k_{0}\right)}{r^{s \gamma}}\right] \\
& \leq \frac{M^{\alpha} 2^{(s+1) \alpha}}{d^{\alpha}} \frac{\varphi^{\beta}\left(k_{0}\right)+\varphi^{\gamma}\left(k_{0}\right)}{r^{s \delta}} \\
& =\frac{\varphi\left(k_{0}\right)}{r^{s+1}}\left[\frac{M^{\alpha} 2^{(s+1) \alpha}}{d^{\alpha}} \frac{\varphi^{\beta-1}\left(k_{0}\right)+\varphi^{\gamma-1}\left(k_{0}\right)}{r^{[s(\delta-1)-1]}}\right] .
\end{aligned}
$$

We choose $r=2^{\frac{\alpha}{\delta-1}}$ and $\frac{M^{\alpha} 2^{\frac{\alpha \delta}{\delta-1}}}{d^{\alpha}}\left(\varphi^{\beta-1}\left(k_{0}\right)+\varphi^{\gamma-1}\left(k_{0}\right)\right) \leq 1$. Consequently, these choices guarantee $\varphi\left(k_{s+1}\right) \leq \frac{\varphi\left(k_{0}\right)}{r^{s+1}}$. From (5.2) and $\varphi(t)$ is nonnegative and nonincreasing, we have deduced the result.

\section{Competing interests}

The authors declare that they have no competing interests.

\section{Authors' contributions}

All authors contributed equally to the manuscript and approved the final version.

\section{Acknowledgements}

The authors would like to thank the anonymous referees for their valuable comments on and suggestions regarding the original manuscript. This work was supported by National Science Foundation of China (11271154), by Key Lab of Symbolic Computation and Knowledge Engineering of Ministry of Education and by the 985 program of Jilin University.

\section{Received: 19 October 2013 Accepted: 20 March 2014 Published: 02 Apr 2014}

\section{References}

1. Rǔžička, M: Electrorheological Fluids: Modeling and Mathematical Theory. Lecture Notes in Mathematics, vol. 1748. Springer, Berlin (2000)

2. DiBenedetto, E: Degenerate Parabolic Equations. Universitext. Springer, New York (1993)

3. Fragnelli, G, Nistri, P, Papini, D: Non-trivial non-negative periodic solutions of a system of doubly degenerate parabolic equations with nonlocal terms. Discrete Contin. Dyn. Syst. 31, 35-64 (2011)

4. Ladyženskaja, OA, Solonnikov, VA, Ural'ceva, NN: Linear and Quasilinear Equations of Parabolic Type. Translations of Mathematical Monographs, vol. 23. Am. Math. Soc., Providence (1968) Translated from the Russian by S. Smith

5. Sun, J, Yin, J, Wang, Y: Asymptotic bounds of solutions for a periodic doubly degenerate parabolic equation. Nonlinear Anal. 74, 2415-2424 (2011)

6. Tsutsumi, M: On solutions of some doubly nonlinear degenerate parabolic equations with absorption. J. Math. Anal. Appl. 132, 187-212 (1988)

7. Wang, J, Gao, W: Existence of nontrivial nonnegative periodic solutions for a class of doubly degenerate parabolic equation with nonlocal terms. J. Math. Anal. Appl. 331, 481-498 (2007)

8. Allegretto, W, Nistri, P: Existence and optimal control for periodic parabolic equations with nonlocal terms. IMA J. Math. Control Inf. 16, 43-58 (1999)

9. Huang, $R$, Wang, Y, Ke, Y: Existence of non-trivial nonnegative periodic solutions for a class of degenerate parabolic equations with nonlocal terms. Discrete Contin. Dyn. Syst., Ser. B 5, 1005-1014 (2005)

10. Nakao, M: Periodic solutions of some nonlinear degenerate parabolic equations. J. Math. Anal. Appl. 104, 554-567 (1984)

11. Pang, PYH, Wang, Y, Yin, J: Periodic solutions for a class of reaction-diffusion equations with $p$-Laplacian. Nonlinear Anal., Real World Appl. 11, 323-331 (2010) 
12. Diening, L, Harjulehto, P, Hästö, P, Rưžička, M: Lebesgue and Sobolev Spaces with Variable Exponents. Lecture Notes in Mathematics, vol. 2017. Springer, Heidelberg (2011)

13. Fragnelli, G: Positive periodic solutions for a system of anisotropic parabolic equations. J. Math. Anal. Appl. 367, 204-228 (2010)

14. Guo, B, Gao, W: Study of weak solutions for parabolic equations with nonstandard growth conditions. J. Math. Anal. Appl. 374, 374-384 (2011)

15. Wu, Z, Yin, J, Wang, C: Elliptic \& Parabolic Equations. World Scientific, Hackensack (2006)

16. Simon, J: Compact sets in the space $L^{P}(0, T ; B)$. Ann. Mat. Pura Appl. (4) 146, 65-96 (1987)

17. Porzio, MM, Vespri, V: Hölder estimates for local solutions of some doubly nonlinear degenerate parabolic equations. J. Differ. Equ. 103, 146-178 (1993)

10.1186/1687-2770-2014-77

Cite this article as: Li and Gao: Existence of nonnegative nontrivial periodic solutions to a doubly degenerate parabolic equation with variable exponent. Boundary Value Problems 2014, 2014:77

Submit your manuscript to a SpringerOpen ${ }^{\circ}$ journal and benefit from:

- Convenient online submission

- Rigorous peer review

- Immediate publication on acceptance

- Open access: articles freely available online

- High visibility within the field

- Retaining the copyright to your article

Submit your next manuscript at $>$ springeropen.com 